\title{
Neighborhood built environment and cognition in non-demented older adults: The Multi-Ethnic Study of Atherosclerosis
}

\author{
Lilah M. Besser, PhD ${ }^{\mathrm{a}}$, Daniel A. Rodriguez, $\mathrm{PhD}^{\mathrm{b}}$, Noreen McDonald, $\mathrm{PhD}^{\mathrm{c}}$, Walter A \\ Kukull, $\mathbf{P h D}^{\mathrm{d}}$, Annette Fitzpatrick, $\mathbf{P h D}^{\mathrm{e}}$, Stephen R. Rapp, $\mathbf{P h D}^{\mathrm{f}}$, and Teresa Seeman, $\mathbf{P h D}{ }^{\mathrm{g}}$ \\ aNational Alzheimer's Coordinating Center, Department of Epidemiology, University of

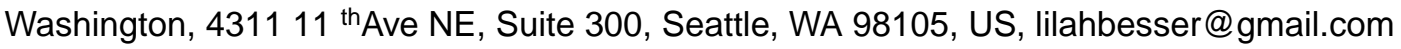 \\ ${ }^{b}$ Department of City and Regional Planning, Institute for Transportation Studies, University of \\ California, Berkeley, Office 313B, Wurster Hall \#1820, Berkeley, California, 94720, US, \\ danrod@berkeley.edu \\ 'Department of City and Regional Planning, University of North Carolina at Chapel Hill, $317 \mathrm{New}$ \\ East Building, CB 3140, Chapel Hill, NC 27599, US, noreen@unc.edu \\ dNational Alzheimer's Coordinating Center, Department of Epidemiology, University of \\ Washington, $431111^{\text {th }}$ Ave NE, Suite 300, Seattle, WA 98105, US, kukull@uw.edu \\ eDepartments of Family Medicine, Epidemiology and Global Health, University of Washington, \\ 1959 NE Pacific Street, Health Sciences Building, F-350, Box 357230, Seattle, WA 98195, US, \\ fitzpal@uw.edu \\ fDepartment of Psychiatry and Behavioral Medicine, Wake Forest School of Medicine, Medical \\ Center Blvd, Winston-Salem, NC 27157, US, srapp@wakehealth.edu \\ 9Department of Medicine, Division of Geriatrics, University of California, Los Angeles, 10945 Le \\ Conte Avenue, Suite 2339, Los Angeles, CA 90095, US, TSeeman@mednet.ucla.edu
}

\begin{abstract}
Preliminary studies suggest that neighborhood social and built environment (BE) characteristics may affect cognition in older adults. Older adults are particularly vulnerable to the neighborhood environment due to a decreasing range of routine travel with increasing age. We examined if multiple neighborhood $\mathrm{BE}$ characteristics are cross-sectionally associated with cognition in a diverse sample of older adults, and if the BE-cognition associations vary by individual-level demographics. The sample included 4,539 participants from the Multi-Ethnic Study of Atherosclerosis. Multivariable linear regression was used to examine the associations between five BE measures and four cognitive measures, and effect modification by individual-level education and race/ethnicity. In the overall sample, increasing social destination density, walking destination density, and intersection density were associated with worse overall cognition, whereas increasing proportion of land dedicated to retail was associated with better processing speed. Effect modification results suggest that the association between urban density and worse cognition may
\end{abstract}

Corresponding author: Lilah Besser, $431111^{\text {th }}$ Ave NE, Suite 300, Seattle, WA 98105, Phone: 206-616-5759, Fax: 206-616- 5927, lilahbesser@gmail.com. 
be limited to or strongest in those of non-white race/ethnicity. Although an increase in neighborhood retail destinations was associated with better cognition in the overall sample, these results suggest that certain BE characteristics in dense urban environments may have a disproportionately negative association with cognition in vulnerable populations. However, our findings must be replicated in longitudinal studies and other regional samples.

\section{Keywords}

neighborhood; built environment; cognition; older adult; cognitive; race; ethnicity; education

\section{Introduction}

Cognitive impairment, present in $\geq 10 \%$ of adults 65 years and older (Unverzagt et al., 2001), is associated with lower quality of life (Muangpaisan et al., 2008) and increased nursing home placement (Gaugler et al., 2007). The impending rise in the population of older adults (US Census) will be accompanied by an increase in the prevalence of cognition impairment, calling for strategies to address the associated economic, health, and social burden. Interventions focused on improving diet and reducing vascular risks may simultaneously delay the onset of cognitive impairment (Nelson \& Tabet, 2015). Additionally, there is emerging recognition that residential environments are important in shaping health behaviors and health outcomes (Koohsari et al., 2015; Sallis et al., 2009). For example, lower neighborhood socioeconomic status has been associated with worse cognition in older adults in previous studies (Clarke et al., 2012). Older adults may be particularly influenced by their neighborhood environment due to a smaller range of routine travel and thus increased exposure to proximal environments (Marottoli et al., 2000). Therefore, policies that promote a safe and walkable neighborhood environment may help older adults age in place and delay the onset of cognitive impairment by providing an environment that is socially and mentally engaging (Cassarino \& Setti, 2015) and supportive of a healthy lifestyle (Clarke et al., 2012).

The neighborhood built environment (BE) comprises all of the physical aspects of the environment (Oxford University Press) surrounding the home, including the road network, buildings, sidewalks and bike paths, parks and public spaces, and amenities such as lighting. The mechanisms by which the neighborhood BE affects cognition are likely complex and multifaceted, and thus the direction of the associations may depend on individual-level characteristics and the BE features under consideration. BE-cognition associations have been explored little to date, with prior studies providing little explication on likely mechanism(s) for any observed associations. Below we outline a number of causal mechanisms, each of which may individually or jointly help explain associations between a specific BE characteristic and cognition.

The neighborhood BE may influence health behaviors such as physical activity (PA) and diet, factors that have been associated with cognition (Boone-Heinonen et al., 2011; Groot et al., 2016). Additionally, urban environments may be associated with increased vehicular pollutant exposure due to decreased distances to busy roadways (Buonocore et al., 2009) and 
decreased air ventilation created by buildings (Yuan et al., 2014). Airborne pollutants (Power et al., 2011) have been associated with worse cognition and brain structure in older adults. Neighborhoods with more social engagement opportunities may improve well-being and decrease stress, anxiety, and depression, consequently improving cognition. On the other hand, neighborhood psychosocial disorder (e.g., crime, graffiti), fear of falls , and sensory overload (e.g., confusing spaces, noise, crowds) may increase social isolation (Aneshensel et al., 2011; James, 2009) and negatively impact cognition if residents minimize time spent in the neighborhood. In addition, neighborhood BE factors such as land use mix, population density, traffic, and noise may improve or worsen quality of life and associated mental health outcomes (e.g., stress) (Fassio et al., 2013; Sarmiento et al., 2010; Stansfeld et al., 1996), thereby affecting cognition. Stress in late-life has been associated with worse cognition in older adults (Aggarwal et al., 2014) and a decrease in stressors has been associated with improved cognition (Dickinson et al., 2011). Lastly, neighborhood BEs may provide cognitive stimulation, which can either improve cognition or cause cognitive overload that worsens cognition. Living in a complex neighborhood environment in older age may provide mental stimulation that helps delay cognitive decline by requiring constant but passive adaptation (Cassarino \& Setti, 2015). However, the neighborhood BE may cause cognitive overload (Lindenberger et al., 2000) among those with physical or mental disabilities or cognitive impairment.

The few published studies on the BE and cognition in older adults found associations between cognition and the presence of a community center or transit stop, condition of public spaces, distance to community resources, street connectivity, land use mix, and area dedicated to the natural environment (Besser et al., 2017; Wu et al., 2017). Some but not all of these studies suggest a positive association between increasing urban density and better cognitive functioning. However, the types of BE and cognitive measures used and methods of defining neighborhoods differed markedly in the studies, and additional work is needed to narrow down the $\mathrm{BE}$ features that may have the greatest influence on cognition, to examine potential effect modifiers, and to investigate associations in diverse samples.

In this study, we examine if five neighborhood BE characteristics representing increased density, street accessibility, and land use mix, typically consistent with increasing urban density/less sprawl (Smart Growth America), are associated with better cognition. We additionally aimed to investigate if the BE-cognition associations vary by individual-level education or race/ethnicity, characteristics previously found to modify the association between neighborhood SES and health (Merkin et al., 2009; Wight et al., 2006). We focused on density of social destinations, walking destinations, and intersections, as well as proportion of land dedicated to residences and retail, because similar measures have been associated with walking in older adults (Cerin et al., 2013; Hall \& McAuley, 2010; Li et al., 2005; Michael et al., 2006; Troped et al., 2017), aiming to investigate characteristics that are simultaneously associated with PA and cognition. Urban planners consider diverse implications of plans and policies, including economic, social, environmental, and healthrelated considerations. Therefore, it will be useful for studies to narrow down specific BE characteristics that may benefit multiple aspects of health (e.g., PA and cognition), to strengthen arguments for future plans and policies aimed at improving health. 


\section{Materials and Methods}

\section{Sample}

The analytic sample originated from 4,716 participants who completed Exam 5 (2010-2012) of the Multi-Ethnic Study of Atherosclerosis (MESA), a longitudinal, population-based cohort study of subclinical cardiovascular disease. MESA has completed five exams since 2000, with a sixth exam currently underway. Participants aged 45- to 84-years-old were enrolled from six US regions (Forsyth County, North Carolina; New York, New York; Baltimore, Maryland; St. Paul, Minnesota; Chicago, Illinois; Los Angeles, California) and individuals of African American, Chinese, and Hispanic race/ethnicity were oversampled. Details about MESA have been published previously (Bild et al., 2002). The final sample excluded those ( $\mathrm{n}=357$ ) who: 1 ) were missing all cognitive test scores; 2 ) were missing all BE measures; 3) were taking Alzheimer's disease medication at any exam (acetylcholinesterase inhibitors or N-methyl-D asparate receptor blocker); 4) had an International Classification of Disease (ICD) code suggesting dementia in death certificate and hospitalization records (Fujiyoshi et al., 2016); or 5) had a Cognitive Abilities Screening Instrument (CASI) score <20, which lacks face validity.

\section{Cognitive measures}

MESA's Exam 5 was the only available exam that included cognitive measures (Fitzpatrick et al., 2015). The cognitive tests included: 1) the CASI (Teng et al., 1994) (version 2), a brief test of global cognition (range: 0-100); 2 and 3) Digit Span Forward (DSF) and Backward (DSB) (Wechsler Adult Intelligence Scale subtests [WAIS-III] (Wechsler, 1997)) (ranges: 016, 0-14, respectively), measures of attention, short term and working memory; and 4) Digit Symbol (DS; subtest of WAIS-III (Wechsler, 1997)), a measure of processing speed (range: 0-133). For the regression analyses, z-scores were calculated for each neuropsychological test by subtracting an individual's score from the entire sample's mean score and dividing the difference by the entire sample's standard deviation. The BE may influence certain aspects of cognition more than others (e.g., processing speed versus short-term memory), and therefore, each of the four cognitive tests were included separately in our analyses because they capture different cognitive domains.

\section{Built environment measures}

The neighborhood measures were originally developed as part of the MESA Neighborhood Study (Diez Roux et al., 2016). Land parcels for each study site were classified as residential (e.g., family homes, apartment complexes/condominiums) or retail (e.g., shopping centers, clothing stores), and the percent of the 1/4-mile, 1/2-mile, and 1-mile buffers dedicated to residences or retail was calculated by dividing the residential/retail area by the total buffer area (Rodríguez et al., 2009). Intersection density was determined by dividing intersection counts (excluding culs-de-sac) for the 1/4-mile, 1/2-mile, and 1-mile buffer by the total buffer area. The densities of social engagement (e.g., beauty shops/barbers, performance-based entertainment) and walking destinations (e.g., postal services, non-beverage eating/dining places) per square mile were calculated for the $1 / 4$-mile, $1 / 2$-mile, and 1-mile area around the home using 2010 National Establishment Time Series (NETS) business data. Neighborhood SES, based on US Census American Community Survey data (2007-2011) at the tract-level, 
was previously developed from a principal components analysis of the percent of neighborhood residents with a bachelor's degree, a high school degree, a managerial occupation, and an annual household income $>\$ 50,000$, and the neighborhood's median home value, median household income, and percent rental income.

\section{Participant characteristics}

Baseline characteristics included age, sex, education, race/ethnicity, marital status, family income, and $\geq 1$ apolipoprotein $\varepsilon 4$ allele (APOE $\varepsilon 4$ ), a genetic risk factor for Alzheimer's disease. We described certain health indicators and conditions, including depression (Center for Epidemiologic Studies Depression Scale [CES-D] score $\geq 16$ ), self-reported diabetes, and medication use for hypertension, hypercholesterolemia, and depression.

\section{Statistical methods}

The sample's demographics, clinical characteristics and APOE genotype were detailed using descriptive statistics. Means and standard deviations (SD), ranges, and the $25^{\text {th }}, 50^{\text {th }}$, and $75^{\text {th }}$ percentiles of the BE measures were calculated, and Pearson correlation coefficients were calculated to examine the correlation between BE measures.

Unadjusted and adjusted linear regression models with generalized estimating equations (accounting for clustering by study site) were employed to examine the BE and cognition associations and effect modification by education and race/ethnicity. Twenty models were run to examine each $\mathrm{BE}$ measure (independent variable) and cognitive test (dependent variable) combination. Results are reported in separate tables for density/street accessibility measures (walking destination, social destination, and intersection density) and for land use measures (proportion of land dedicated to residences or retail) for ease of readability. Multiple BE measures were highly correlated in our sample; however, may not necessarily be highly correlated in other samples or neighborhoods/regions. Thus, we chose to evaluate the BE measures separately. Additionally, we aimed to avoid a composite BE measure (e.g., sprawl index) that would limit the specificity in interpreting the results and the ability to contribute to evidence suggesting specific BE features to be targeted in future plans and policies. The $\mathrm{BE}$ and cognitive measures were treated as continuous variables, and the multivariable models adjusted for age, sex, race/ethnicity, income, marital status, neighborhood SES, and $\geq 1$ APOE $\varepsilon 4$ allele.

Interaction terms (e.g., intersection densityxeducation) were entered into the multivariable models to test for effect modification by education ( $₫ 2$ years versus $>12$ years) or race/ ethnicity (Chinese-American, African-American, and Hispanic, versus non-Hispanic white). Our reporting of results focuses on statistically significant interactions $(p<0.05)$ with statistically significant $(\mathrm{p}<0.05)$ associations in $\geq 1$ of the stratified groups (e.g., among Hispanics).

The $1 / 2$-mile buffers around the participants' homes were hypothesized to be the area most representative of older adult neighborhoods (i.e., a reasonable walking distance), and therefore were the primary focus. However, for the main effects analyses, we also evaluated the 1/4-mile and 1-mile BE measures to assess consistency across different buffer sizes. 


\section{Results}

The final sample included 4,539 participants. The majority was 55- to 84-years of age, female, college educated, and married (Table 1). Forty-one percent were non-Hispanic whites, 12\% Chinese-American, 27\% African-American, and 21\% Hispanic. Twenty-seven percent were APOE $\varepsilon 4$ carriers and $14 \%$ had depression. The mean cognitive test scores were 87.8 for the CASI, 9.7 for the DSF, 5.6 for the DSB, and 50.8 for the DS (Table 2). In addition to the results presented in the main tables, additional data and sensitivity analyses are provided in the supplemental material.

\section{Main effects analyses}

In the unadjusted analyses, increasing social destination density was associated with better CASI scores and increasing social and walking destination densities were associated with better DSB scores (see Appendix). Lastly, increasing social destination density was associated with better DS scores.

In the adjusted analyses, compared to those living in neighborhoods with the lowest social and walking destination densities, those living in the highest social and walking destination densities in the $1 / 2$-mile surrounding the home scored worse on the CASI by 0.33 and 0.29 SDs, respectively (Table 3). Compared to those living in neighborhoods with the lowest intersection densities, individuals living in the highest intersection densities in the $1 / 2$-mile surrounding the home scored worse on the CASI by $0.25 \mathrm{SD}$. Although the magnitude of the estimates changed when examining these same associations using the 1/4-mile and 1-mile BE measures, the associations were consistently in the same direction (increasing BE measure associated with worse cognition) and were all statistically significant (Table 3). Additionally, an increasing walking destination density in the 1/4-mile surrounding the home (but not the $1 / 2$-mile or 1-mile) was associated with worse scores on the DSB test.

The results were suggestive of an association between proportion of land dedicated to retail and cognition, although only observed using the $1 / 4$-mile measure (Table 4 ). Compared to those living in neighborhoods with the lowest proportion of land dedicated to retail, individuals living in neighborhoods with the highest proportion of land dedicated to retail scored better on the DS by $0.13 \mathrm{SD}$.

\section{Effect modification by education}

Education modified the association between social destination density and cognition (Table 5). Increasing social destination density was associated with worse DSF scores in those with low education but not in those with high education. Associations between proportion of land dedicated to residences/retail and cognition did not vary by education (Table 6).

\section{Effect modification by race/ethnicity}

Race/ethnicity modified numerous BE-cognition associations (Tables 7 and 8). The associations between three $\mathrm{BE}$ measures and cognition varied significantly when comparing Chinese and non-Hispanic white participants. Increasing social destination density was associated with worse DSB scores, whereas increasing intersection density was associated 
with better CASI and DS scores in Chinese but not non-Hispanic white participants (Table 7). In addition, increasing proportion of land dedicated to retail was associated with worse DSB scores in Chinese but not non-Hispanic white participants (Table 8).

The associations between three BE measures and cognition varied significantly when comparing African American and non-Hispanic white participants. Increasing social destination density was associated with worse DSF and DSB scores and increasing walking destination density was associated with worse DSF scores in African Americans, associations for the most part not observed in non-Hispanic whites (Table 7). Similarly, increasing proportion of land dedicated to residences was associated with better CASI scores in non-Hispanic whites but not in African Americans (Table 8).

The associations between all five BE measures and cognition varied significantly when comparing Hispanic and non-Hispanic white participants. Increasing social destination density was associated with significantly worse CASI and DS scores, and increasing walking destination density and intersection density were associated with worse CASI, DSB, and DS scores in Hispanics (Table 7). No associations were observed between these same BE and cognitive measures among non-Hispanic whites. Also in Hispanics, increasing proportion of land dedicated to residences was associated with better DSB and DS scores and increasing proportion of land dedicated to retail was associated with worse DS scores, with no such associations observed for non-Hispanic whites (Table 8).

\section{Conclusions}

This study provides cross-sectional evidence for an association between the neighborhood $\mathrm{BE}$ and cognition in older adults, independent of individual-level demographics and neighborhood-level SES. Unexpectedly, increasing social destination density, walking destination density, and intersection density were associated with worse cognition in the overall sample and more noticeably in individuals of non-white race. The exception to this pattern was the association between increasing proportion of land dedicated to retail and better cognition in the overall sample. However, the potentially beneficial association of more retail destinations in the neighborhood on cognition appeared to be limited to nonHispanic whites and African American participants, as it was associated with significantly worse cognition in Chinese and Hispanic participants.

Although increasing densities of social and walking destinations were associated with worse cognition in this study, the results from past studies examining similar measures and cognition have been mixed. One study found that access to a community center was associated with slower cognitive decline (Clarke et al., 2015), another found that closer access to community resources (e.g., grocery store) was associated with worse cognition (Magaziner \& Cadigan, 1989), while another found no association between presence of recreation centers and institutions in the neighborhood and cognition (Clarke et al., 2012). It was initially hypothesized that increased access to neighborhood social and walking destinations would be associated with improvements in cognition by improving PA levels, social engagement, mental health, or quality of life. The unexpected findings in this study may be due to residual confounding by unmeasured factors, increased exposure to air 
pollution due to increased walking in the neighborhood which worsens cognition, or factors related to study design (e.g., cross-sectional nature). Thus, additional research on the topic is necessary.

The negative relationship between intersection density and cognition in this study is comparable to findings from another study that used a different measure of street connectivity (i.e., integration) (Watts et al., 2015). The authors speculated that greater integration (less navigational turns to reach a given destination) may create cognitive overload among older adults because of the greater number of initial choices, or may induce stress when walking due to the associated higher levels of traffic. In that previous study, a separate measure of street connectivity (number paths/streets connected to a given street in the network) was associated with less cognitive decline, which the authors posited to be indicative of the benefits of increased accessibility or availability of walking or social destinations. However, increased accessibility to walking and social destinations was associated with worse cognition in our study. While our results were surprising and contrary to our hypothesis, neighborhoods with higher intersection densities may be associated with unmeasured aspects of the environment that negatively impact cognition, such as traffic, noise, or air pollution. Additional studies are needed to assess whether accessibility/street connectivity is negatively and causally related to cognition.

An increased proportion of the neighborhood dedicated to retail had a positive association with cognition. No other known studies have examined this particular measure in relation to cognition; however, two studies found that increased land use mix was associated with lower odds of cognitive impairment and dementia (Wu et al., 2017; Wu et al., 2015). It is possible that unlike other BE characteristics often consistent with increasing urban density, the availability of more retail destinations specifically may promote increased utilitarian physical activity or social engagement that is then associated with improved cognition. In addition or alternatively, increased retail availability may encourage spending time in the neighborhood, which may increase cognitively stimulating activity or may be associated with improved quality of life overall, thereby improving cognition. This is speculative, as much more work is needed to determine if the associations are causal and the underlying mechanisms to explain them.

The associations between increasing social destination density and worse cognition was only observed in those with low education and not those with higher levels of education. Two previous studies have demonstrated similar interactions between neighborhood characteristics and individual-level education in relation to cognitive outcomes (Aneshensel et al., 2011; Wight et al., 2006). The authors found that the associations between lower neighborhood SES and worse cognition were strongest in those with lower education levels. Considering our findings together with those previous studies, individuals of lower SES may be more vulnerable to any possible negative effects on cognition from neighborhood exposures typically consistent with increasing urban density. However, it must be noted that individual-level education did not modify most of the BE-cognition associations in this study. Other measures of individual-level SES were limited in the MESA dataset, and thus investigation of effect modification by other SES measures is called for in future studies. 
BE characteristics often associated with increasing urban density may differentially impact individuals of non-white race. We found that Chinese, African American, and Hispanic participants had worse cognition if they lived in neighborhoods with greater social destination, walking destination, and intersection densities. Additionally, we found that living in neighborhoods with more retail destinations was associated with worse cognition in Chinese and Hispanic participants. At least two previous studies had relevant findings. In one study, Mexican Americans living in barrios (typically higher density) had worse cognition compared to Hispanics living in suburban neighborhoods (Espino et al., 2001). The other study found that presence of institutional resources in the neighborhood was associated with worse cognition in African Americans but better cognition among whites (Clarke et al., 2012). The associations in our study were found after controlling for individual-level and neighborhood-level SES, suggesting that other factors related to nonwhite race/ethnicity may increase vulnerability to the potentially harmful effects of increasing urban density on cognition.

Although at least one of the BE measures consistent with increasing urban density was associated with better processing speed among non-Hispanic white, Chinese, and African American participants, the same was not true for Hispanic participants. In addition, a large percentage of the associations with worse cognition were found for Hispanics versus the other races/ethnicities. Unlike the non-Hispanic whites who were almost all US born and spoke English as their primary language, $65 \%$ of Hispanics were foreign born and approximately half spoke Spanish as their primary language (data not shown). Among whites, certain BE characteristics may be associated with improvements in cognition through improvements in PA. In contrast, among Hispanics immigrants, a compact BE may have an overall negative effect if unfamiliar cultures or languages in the neighborhood cause cognitive overload. Alternatively, other unknown factors associated with Hispanic enclaves may help explain our observed associations. For instance, the associations between dense urban environments and worse cognition may be explained by lower levels of acculturation among Hispanics. We did not further investigate ethnic enclaves/acculturation in our analyses because the available measures on acculturation/primary language were highly correlated with race/ethnicity. New studies of the BE and cognition that are focused specifically on Hispanics will be the best positioned to address the potential impact of acculturation.

While outside the scope of our study, we quickly examined available data on food environments. Although the mean density of unfavorable food stores was similar between Hispanics and non-Hispanic whites, the median and $75^{\text {th }}$ percentile were higher for Hispanics. It is possible that one or more of the BE measures included in our study were partial proxies for unfavorable food environments. Greater availability of fast food options has been associated with increased fast food consumption in younger adults (BooneHeinonen et al., 2011). In turn, diabetes, a diet-related health condition, has been associated with brain atrophy and cognitive impairment (Roberts et al., 2014). Food environments as an inherent part of the BE may be associated with changes in diet, a risk factor previously demonstrated to influence cognition in older adults. Therefore, further examination of associations between neighborhood food environments and cognition may be a fruitful avenue for future research that may help explain our observed disparities. 
The causal mechanisms to explain observed associations are unclear, and as one of the first to examine BE-cognition associations, this study was not expected to teasing apart the potential mechanisms. However, we observed associations with each of the four cognitive tests, suggesting that the neighborhood BE may affect multiple aspects of cognition, including attention, short-term and working memory, and processing speed. The association between increasing urban density and better processing speed may be explained by the need for greater cognitive processing of complex urban environments, or could also be related to increased levels of overall PA in dense urban environments. Typically, processing speed slows with age and is associated with less white matter integrity in the brain (Albinet et al., 2012). In turn, white matter integrity is better preserved among older adults obtaining more PA (Tian et al., 2015). The negative associations may be explained by increased access to unhealthy foods, stress, or psychosocial responses (e.g., fear of others) that can accompany increasing urban density, which may decrease healthy behaviors or quality of life, worsening cognition. Overall, our study suggests differing associations between the $\mathrm{BE}$ and cognition based on the cognitive domain examined. Specific aspects of the BE may affect only specific cognitive domains, and keeping these measures as explicit as possible in future studies may help elucidate the causal mechanisms.

The strengths of this study included the use a multi-ethnic, multi-site cohort recruited through population-based methods, which improves the generalizability of the findings. MESA provides a rich source of demographic, clinical, and neighborhood data that allowed for the control of important confounders. Additionally, when the 1/4- and 1-mile BE measures were used instead of the 1/2-mile measures, the findings changed in some instances but were generally similar in the direction of the association, suggesting that the findings are relatively robust regardless of the neighborhood scale (i.e., buffer size) used.

Nevertheless, this study has limitations, first and foremost its cross-sectional nature. Our results must be replicated in other cohorts and using methods that consider longitudinal measures of the BE and cognition to provide evidence for a causal association. We were not able to account for bias due to neighborhood self-selection, in which preferences for moving to a particular BE may also be related to an individual's cognition or factors associated with cognition (James et al., 2015). However, the large majority of MESA participants did not move since their baseline exam (Hirsch et al., 2014) and almost half did not move during the 20 years preceding MESA enrollment (Murray et al., 2010), consistent with the expectation of decreased residential mobility with age (Plane et al., 2005). There is some evidence that the MESA participants tended to move between neighborhoods with similar SES levels (Murray et al., 2010), and future research should examine whether this pattern can be extrapolated to neighborhood BE characteristics. The inconsistency of findings between our study and past studies may relate to differences in the definitions of the BE characteristics, the neighborhood scales (e.g., 1/2-mile surrounding home versus US Census tract), the cognitive measures used, the study designs (e.g., longitudinal or cross-sectional), or the sample selection. Additionally, attrition since enrollment into the study may have affected our findings, as those who remained in MESA as of the Exam 5 were more often of higher SES, married, and non-Hispanic white (data not shown). Finally, unmeasured aspects of the neighborhood or residual confounding by individual-level factors may have biased our results. Future research must carefully consider the individual-level and neighborhood-level 
characteristics that are likely mediators versus confounders of the BE-cognition association, as well as which characteristics are more likely to be independent predictors of cognition and therefore not confounders. An example would be neighborhood social characteristics, which in this study were hypothesized to be either mediators or independent predictors of cognition but not confounders of the BE-cognition associations, and therefore, were not controlled for in our analyses.

Subsequent studies should employ a more expansive cognitive test battery than available in MESA, to explore other cognitive domains that may be associated with the neighborhood $\mathrm{BE}$ and to help address the limitations the CASI, a brief cognitive test of overall cognition. Although not available for all six MESA sites, neighborhood parks and greenspace could be examined in future studies, as they have been previously associated with increased PA (Sugiyama et al., 2010) and improved mental health (Sturm \& Cohen, 2014), and thus may be associated with cognition. In addition, other BE scales may be important to consider in tandem with the immediately surrounding neighborhood environment, such as the bordering neighborhoods and their availability of social or walking destinations or transit connections. Finally, new studies may benefit from consideration of BE typologies, sets of BE characteristics that typically accompany one another (e.g., higher intersection density is correlated with higher walking destination density), given that their individual-level effects may be difficult to disentangle.

In this study, neighborhood BE characteristics were cross-sectionally associated with cognition in older adults, and many BE-cognition associations varied by race/ethnicity, suggesting these associations are complex and depend on individual-level characteristics. The findings have significant implications for urban planning for equity if replicated in future studies. Compact growth principles, which increase urban density and have been recommended as a way of allowing older adults to age in place, may have detrimental effects on the cognitive functioning of vulnerable populations. Ideally, planners and public health researchers would evaluate compact growth policy implications on diverse populations of older adults, and weigh the potentially positive (e.g., increased PA) and negative consequences to health (e.g., worse cognition). In the process, additional policies and programs could be devised to offset any potential harmful effects of increased urban density on cognition among susceptible individuals. However, before our findings can be incorporated into urban planning considerations, our results must be replicated in longitudinal studies and in other regional samples.

\section{Supplementary Material}

Refer to Web version on PubMed Central for supplementary material.

\section{References}

Aggarwal NT, Wilson RS, Beck TL, Rajan KB, Mendes de Leon CF, Evans DA, et al. Perceived stress and change in cognitive function among adults 65 years and older. Psychosom Med. 2014; 76:8085. [PubMed: 24367123] 
Albinet CT, Boucard G, Bouquet CA, Audiffren M. Processing speed and executive functions in cognitive aging: how to disentangle their mutual relationship? Brain Cogn. 2012; 79:1-11. [PubMed: 22387275]

Aneshensel CS, Ko MJ, Chodosh J, Wight RG. The urban neighborhood and cognitive functioning in late middle age. J Health Soc Behav. 2011; 52:163-179. [PubMed: 21673145]

Besser LM, McDonald NC, Song Y, Kukull WA, Rodriguez DA. Neighborhood Environment and Cognition in Older Adults: A Systematic Review. Am J Prev Med. 2017; doi: 10.1212/WNL. 0000000000004521

Bild DE, Bluemke DA, Burke GL, Detrano R, Diez Roux AV, Folsom AR, et al. Multi-Ethnic Study of Atherosclerosis: objectives and design. Am J Epidemiol. 2002; 156:871-881. [PubMed: 12397006]

Boone-Heinonen J, Gordon-Larsen P, Kiefe CI, Shikany JM, Lewis CE, Popkin BM. Fast food restaurants and food stores: longitudinal associations with diet in young to middle-aged adults: the CARDIA study. Arch Intern Med. 2011; 171:1162-1170. [PubMed: 21747011]

Buonocore JJ, Lee HJ, Levy JI. The influence of traffic on air quality in an urban neighborhood: a community-university partnership. Am J Public Health. 2009; (99 Suppl 3):S629-635. [PubMed: 19890168]

Cassarino M, Setti A. Environment as 'Brain Training': A review of geographical and physical environmental influences on cognitive ageing. Ageing Res Rev. 2015; 23:167-182. [PubMed: 26144974]

Cerin E, Lee KY, Barnett A, Sit CH, Cheung MC, Chan WM, et al. Walking for transportation in Hong Kong Chinese urban elders: a cross-sectional study on what destinations matter and when. Int J Behav Nutr Phys Act. 2013; 10:78. [PubMed: 23782627]

Clarke PJ, Ailshire JA, House JS, Morenoff JD, King K, Melendez R, et al. Cognitive function in the community setting: the neighbourhood as a source of 'cognitive reserve'? J Epidemiol Community Health. 2012; 66:730-736. [PubMed: 21515547]

Clarke PJ, Weuve J, Barnes L, Evans DA, Mendes de Leon CF. Cognitive decline and the neighborhood environment. Ann Epidemiol. 2015; 25:849-54. [PubMed: 26253697]

Cramer V, Torgersen S, Kringlen E. Quality of Life in a City: The Effect of Population Density. Social Indicators Research. 2004; 69:103-116.

Dickinson WJ, Potter GG, Hybels CF, McQuoid DR, Steffens DC. Change in stress and social support as predictors of cognitive decline in older adults with and without depression. Int J Geriatr Psychiatry. 2011; 26:1267-1274. [PubMed: 21370277]

Diez Roux AV, Mujahid MS, Hirsch JA, Moore K, Moore LV. The Impact of Neighborhoods on CV Risk. Glob Heart. 2016; 11:353-363. [PubMed: 27741982]

Espino DV, Lichtenstein MJ, Palmer RF, Hazuda HP. Ethnic differences in mini-mental state examination (MMSE) scores: where you live makes a difference. J Am Geriatr Soc. 2001; 49:538548. [PubMed: 11380745]

Fassio O, Rollero C, De Piccoli N. Health, Quality of Life and Population Density: A Preliminary Study on "Contextualized” Quality of Life. Social Indicators Research. 2013; 110:479-488.

Fitzpatrick AL, Rapp SR, Luchsinger J, Hill-Briggs F, Alonso A, Gottesman R, et al. Sociodemographic Correlates of Cognition in the Multi-Ethnic Study of Atherosclerosis (MESA). Am J Geriatr Psychiatry. 2015; 23:684-97. [PubMed: 25704999]

Fujiyoshi A, Jacobs DR, Alonso A, Luchsinger JA, Rapp SR, Duprez DA. Validity of Death Certificate and Hospital Discharge ICD Codes for Dementia Diagnosis: The Multi-Ethnic Study of Atherosclerosis. Alzheimer Dis Assoc Disord. 2016; 31:168-172.

Gaugler JE, Duval S, Anderson KA, Kane RL. Predicting nursing home admission in the U.S: a metaanalysis. BMC Geriatr. 2007; 7:13. [PubMed: 17578574]

Groot C, Hooghiemstra AM, Raijmakers PG, van Berckel BN, Scheltens P, Scherder EJ, et al. The effect of physical activity on cognitive function in patients with dementia: A meta-analysis of randomized control trials. Ageing Res Rev. 2016; 25:13-23. [PubMed: 26607411]

Hall KS, McAuley E. Individual, social environmental and physical environmental barriers to achieving 10000 steps per day among older women. Health Educ Res. 2010; 25:478-488. [PubMed: 20348166] 
Hirsch JA, Moore KA, Clarke PJ, Rodriguez DA, Evenson KR, Brines SJ, et al. Changes in the built environment and changes in the amount of walking over time: longitudinal results from the multiethnic study of atherosclerosis. Am J Epidemiol. 2014; 180:799-809. [PubMed: 25234431]

James, BD. Social engagement and cognitive decline in older adults: Pathways and neighborhood context. Ann Arbor: The Johns Hopkins University; 2009. p. 188

James P, Hart JE, Arcaya MC, Feskanich D, Laden F, Subramanian SV. Neighborhood Self-Selection: The Role of Pre-Move Health Factors on the Built and Socioeconomic Environment. Int J Environ Res Public Health. 2015; 12:12489-12504. [PubMed: 26457712]

Koohsari MJ, Badland H, Sugiyama T, Mavoa S, Christian H, Giles-Corti B. Mismatch between perceived and objectively measured land use mix and street connectivity: associations with neighborhood walking. J Urban Health. 2015; 92:242-252. [PubMed: 25539783]

Li F, Fisher KJ, Brownson RC, Bosworth M. Multilevel modelling of built environment characteristics related to neighbourhood walking activity in older adults. J Epidemiol Community Health. 2005; 59:558-564. [PubMed: 15965138]

Lindenberger U, Marsiske M, Baltes PB. Memorizing while walking: increase in dual-task costs from young adulthood to old age. Psychol Aging. 2000; 15:417-436. [PubMed: 11014706]

Magaziner J, Cadigan DA. Community resources and mental health of older women living alone. J Aging Health. 1989; 1:35-49. [PubMed: 10293789]

Marottoli RA, de Leon CFM, Glass TA, Williams CS, Cooney LM, Berkman LF. Consequences of driving cessation: decreased out-of-home activity levels. J Gerontol B Psychol Sci Soc Sci. 2000; 55:S334-340. [PubMed: 11078110]

Martinez, DM. Racial disparities in cognitive performance over time among older adults: A multilevel analysis of neighborhood effects. Ann Arbor: University of California Los Angeles; 2007.

Merkin SS, Basurto-Dávila R, Karlamangla A, Bird CE, Lurie N, Escarce J, et al. Neighborhoods and cumulative biological risk profiles by race/ethnicity in a national sample of U.S. adults: NHANES III. Ann Epidemiol. 2009; 19:194-201. [PubMed: 19217002]

Michael Y, Beard T, Choi D, Farquhar S, Carlson N. Measuring the influence of built neighborhood environments on walking in older adults. J Aging Phys Act. 2006; 14:302-312. [PubMed: 17090807]

Muangpaisan W, Assantachai P, Intalapaporn S, Pisansalakij D. Quality of life of the community-based patients with mild cognitive impairment. Geriatr Gerontol Int. 2008; 8:80-85. [PubMed: 18713159]

Murray ET, Diez Roux AV, Carnethon M, Lutsey PL, Ni H, O’Meara ES. Trajectories of neighborhood poverty and associations with subclinical atherosclerosis and associated risk factors: the multiethnic study of atherosclerosis. Am J Epidemiol. 2010; 171:1099-1108. [PubMed: 20423931]

Nelson L, Tabet N. Slowing the progression of Alzheimer's disease; what works? Ageing Res Rev. 2015; 23:193-209. [PubMed: 26219494]

Oxford University Press. [[accessed 12/18/2017]] Definition of built environment in English. Available at: https://en.oxforddictionaries.com/definition/built_environment

Plane DA, Henrie CJ, Perry MJ. Migration up and down the urban hierarchy and across the life course. Proc Natl Acad Sci U S A. 2005; 102:15313-15318. [PubMed: 16230633]

Power MC, Weisskopf MG, Alexeeff SE, Coull BA, Spiro A, Schwartz J. Traffic-related air pollution and cognitive function in a cohort of older men. Environ Health Perspect. 2011; 119:682-687. [PubMed: 21172758]

Rodríguez DA, Evenson KR, Diez Roux AV, Brines SJ. Land use, residential density, and walking. The multi-ethnic study of atherosclerosis. Am J Prev Med. 2009; 37:397-404. [PubMed: 19840694]

Sallis JF, Cerin E, Conway TL, Adams MA, Frank LD, Pratt M, et al. Physical activity in relation to urban environments in 14 cities worldwide: a cross-sectional study. Lancet. 2016; 387:2207-2217. [PubMed: 27045735]

Sallis JF, Saelens BE, Frank LD, Conway TL, Slymen DJ, Cain KL, et al. Neighborhood built environment and income: examining multiple health outcomes. Soc Sci Med. 2009; 68:1285-1293. [PubMed: 19232809] 
Sarmiento OL, Schmid TL, Parra DC, Díaz-del-Castillo A, Gómez LF, Pratt M, et al. Quality of life, physical activity, and built environment characteristics among colombian adults. J Phys Act Health. 2010; (7 Suppl 2):S181-195. [PubMed: 20702906]

Smart Growth America. [[accessed 12/18/2017]] Measuring Sprawl. 2014. Available: https:// www.smartgrowthamerica.org/app/legacy/documents/measuring-sprawl-2014.pdf

Stansfeld S, Gallacher J, Babisch W, Shipley M. Road traffic noise and psychiatric disorder: prospective findings from the Caerphilly Study. BMJ. 1996; 313:266-267. [PubMed: 8704537]

Sturm R, Cohen D. Proximity to urban parks and mental health. J Ment Health Policy Econ. 2014; 17:19-24. [PubMed: 24864118]

Sugiyama T, Francis J, Middleton NJ, Owen N, Giles-Corti B. Associations between recreational walking and attractiveness, size, and proximity of neighborhood open spaces. Am J Public Health. 2010; 100:1752-1757. [PubMed: 20634455]

Teng EL, Hasegawa K, Homma A, Imai Y, Larson E, Graves A, et al. The Cognitive Abilities Screening Instrument (CASI): a practical test for cross-cultural epidemiological studies of dementia. Int Psychogeriatr. 1994; 6:45-58. [PubMed: 8054493]

Tian Q, Glynn NW, Erickson KI, Aizenstein HJ, Simonsick EM, Yaffe K, et al. Objective measures of physical activity, white matter integrity and cognitive status in adults over age 80. Behav Brain Res. 2015; 284:51-57. [PubMed: 25655514]

Troped PJ, Tamura K, McDonough MH, Starnes HA, James P, Ben-Joseph E, et al. Direct and Indirect Associations Between the Built Environment and Leisure and Utilitarian Walking in Older Women. Ann Behav Med. 2017; 51:282-291. [PubMed: 27807683]

Unverzagt FW, Gao S, Baiyewu O, Ogunniyi AO, Gureje O, Perkins A, et al. Prevalence of cognitive impairment: data from the Indianapolis Study of Health and Aging. Neurology. 2001; 57:16551662. [PubMed: 11706107]

US Census. [[accessed 08/22/2015]] An Aging Nation: The Older Population in the United States Population Estimates and Projections. Available: https://www.census.gov/prod/2014pubs/ p25-1140.pdf

Watts A, Ferdous F, Moore KD, Burns JM. Neighborhood Integration and Connectivity Predict Cognitive Performance and Decline. Gerontol Geriatr Med. 2015; 1:1-9.

Wechsler, D. Wechsler Adult Intelligence Scale. San Antonio, TX: The Psychological Corporation; 1997.

Wight RG, Aneshensel CS, Miller-Martinez D, Botticello AL, Cummings JR, Karlamangla AS, et al. Urban neighborhood context, educational attainment, and cognitive function among older adults. Am J Epidemiol. 2006; 163:1071-1078. [PubMed: 16707655]

Wu YT, Prina AM, Jones A, Matthews FE, Brayne C. \& MRC-CFAAS Collaboration. The Built Environment and Cognitive Disorders: Results From the Cognitive Function and Ageing Study II. Am J Prev Med. 2017; 53:25-32. [PubMed: 28082001]

Wu YT, Prina AM, Jones AP, Barnes LE, Matthews FE, Brayne C, et al. Community environment, cognitive impairment and dementia in later life: results from the Cognitive Function and Ageing Study. Age Ageing. 2015; 44:1005-1011. [PubMed: 26464419]

Yuan $\mathrm{C}, \mathrm{Ng}$ E, Norford LK. Improving air quality in high-density cities by understanding the relationship between air pollutant dispersion and urban morphologies. Building and Environment. 2014; 71:245-258. 


\section{Table 1}

Demographics and clinical characteristics $(\mathrm{n}=4,359)$

\begin{tabular}{lc}
\hline Characteristic & $\mathbf{n}(\%)$ \\
\hline Age, exam 5 (years) & $73(1.7 \%)$ \\
$45-54$ & $1476(33.9 \%)$ \\
$55-64$ & $1397(32.1 \%)$ \\
$65-74$ & $1148(26.3 \%)$ \\
$75-84$ & $265(6.1 \%)$ \\
285 & $2041(46.8 \%)$ \\
Male & \\
Education & $588(13.5 \%)$ \\
< High school degree & $754(17.3 \%)$ \\
High school degree & $1273(29.3 \%)$ \\
Some college & $1737(39.9 \%)$ \\
Bachelor's degree & $2771(64.2 \%)$ \\
Married & \\
Race/ethnicity & $1777(40.8 \%)$ \\
White/Caucasian & $504(11.6 \%)$ \\
Chinese-American & $1162(26.7 \%)$ \\
Black/African American & $916(21.0 \%)$ \\
Hispanic & $2839(67.6 \%)$ \\
Family income $\$ 30,000 /$ year & $1083(26.5 \%)$ \\
$\geq 1$ APOE 84 allele & $614(14.4 \%)$ \\
Depression (CES-D $\geq 16)$ & $461(10.6 \%)$ \\
Diabetes & $2402(55.18 \%)$ \\
Hypertension medication & $1693(38.8 \%)$ \\
Hypercholesterolemia medication & $333(7.6 \%)$ \\
Cardiovascular disease & $136(3.1 \%)$ \\
\hline
\end{tabular}

Abbreviations: $\mathrm{APOE}=$ apolipoprotein E; CES-D = Center for Epidemiologic Studies Depression scale; TIA = transient ischemic attack Missing: APOE, $\mathrm{n}=268$; income, $\mathrm{n}=156$; education, $\mathrm{n}=7$; CES-D, $\mathrm{n}=86$; diabetes, $\mathrm{n}=26$; married, $\mathrm{n}=42$; cardiovascular disease, $\mathrm{n}=2$; cerebrovascular disease, $\mathrm{n}=2$ 


\section{Table 2}

Cognitive test scores and built environment characteristics

\begin{tabular}{lc}
\hline Measure & $\begin{array}{c}\text { Mean } \pm \text { Standard } \\
\text { deviation }\end{array}$ \\
\hline CASI & $87.8 \pm 8.7$ \\
DSF & $9.7 \pm 2.8$ \\
DSB & $5.6 \pm 2.4$ \\
DS & $50.8 \pm 18.4$ \\
Social destination density & $a$ \\
Walking destination density $^{a}$ & $68.7 \pm 106.2$ \\
Intersection density $^{a}$ & $0.79 \pm 0.52$ \\
Proportion land residential $^{a}$ & $0.47 \pm 0.17$ \\
Proportion land retail $^{a}$ & $0.048 \pm 0.051$ \\
\hline
\end{tabular}

Abbreviations: CASI = Cognitive Abilities Screening Instrument; DSF = Digit Span Forward; DSB = Digit Span Backward; DS = Digit Symbol

Missing: CASI, $\mathrm{n}=8$; DSF, $\mathrm{n}=16$; DSB, $\mathrm{n}=16$; DS, $\mathrm{n}=405$; proportion residential, $\mathrm{n}=283$; proportion retail, $\mathrm{n}=283$

$a_{1 / 2 \text {-mile buffer }}$ 


\section{롤 \\ 꼴}

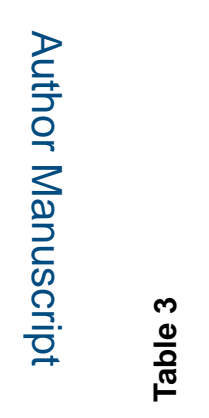

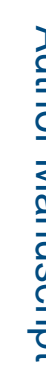

言)

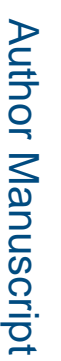

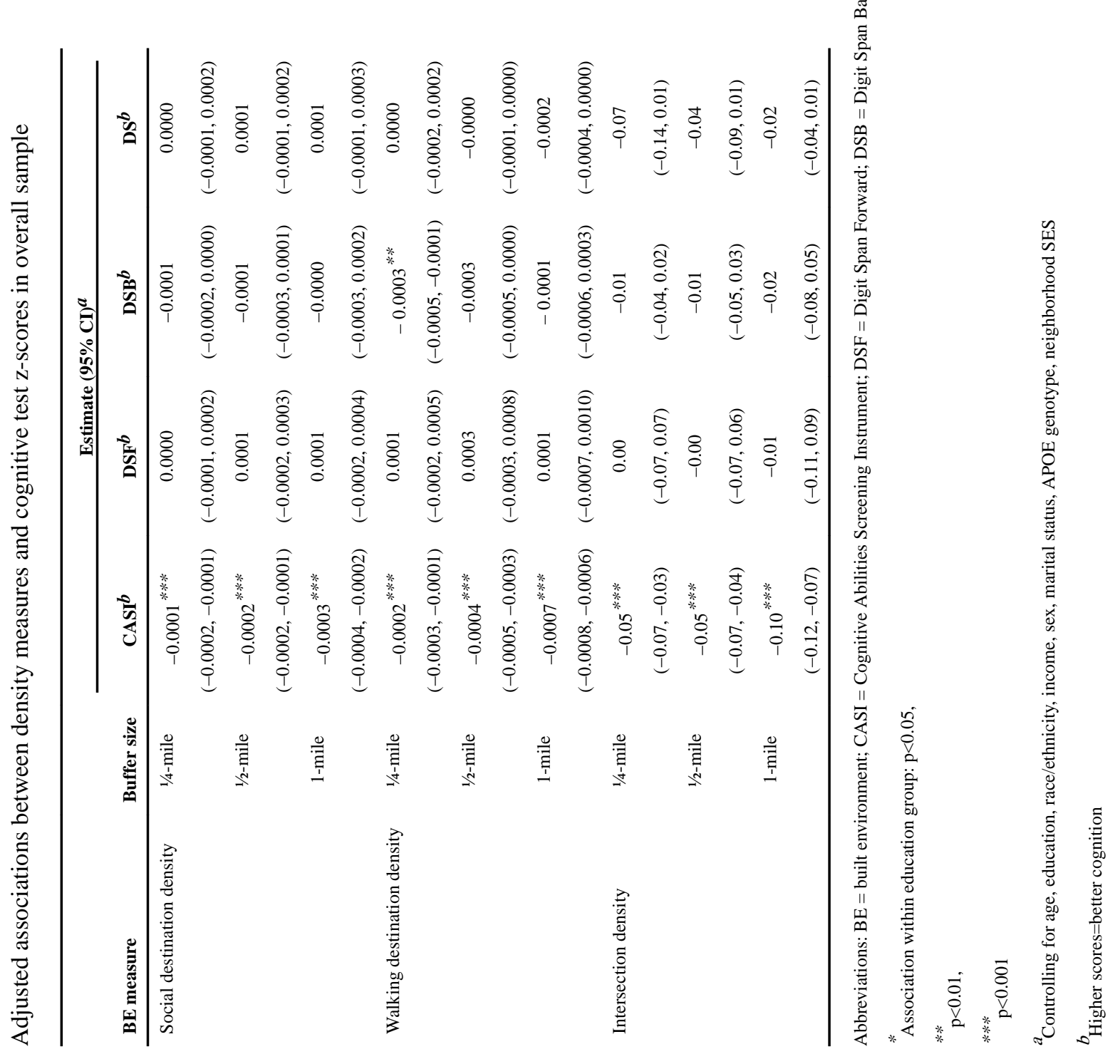

Soc Sci Med. Author manuscript; available in PMC 2019 March 01. 


\section{롤 \\ 길}

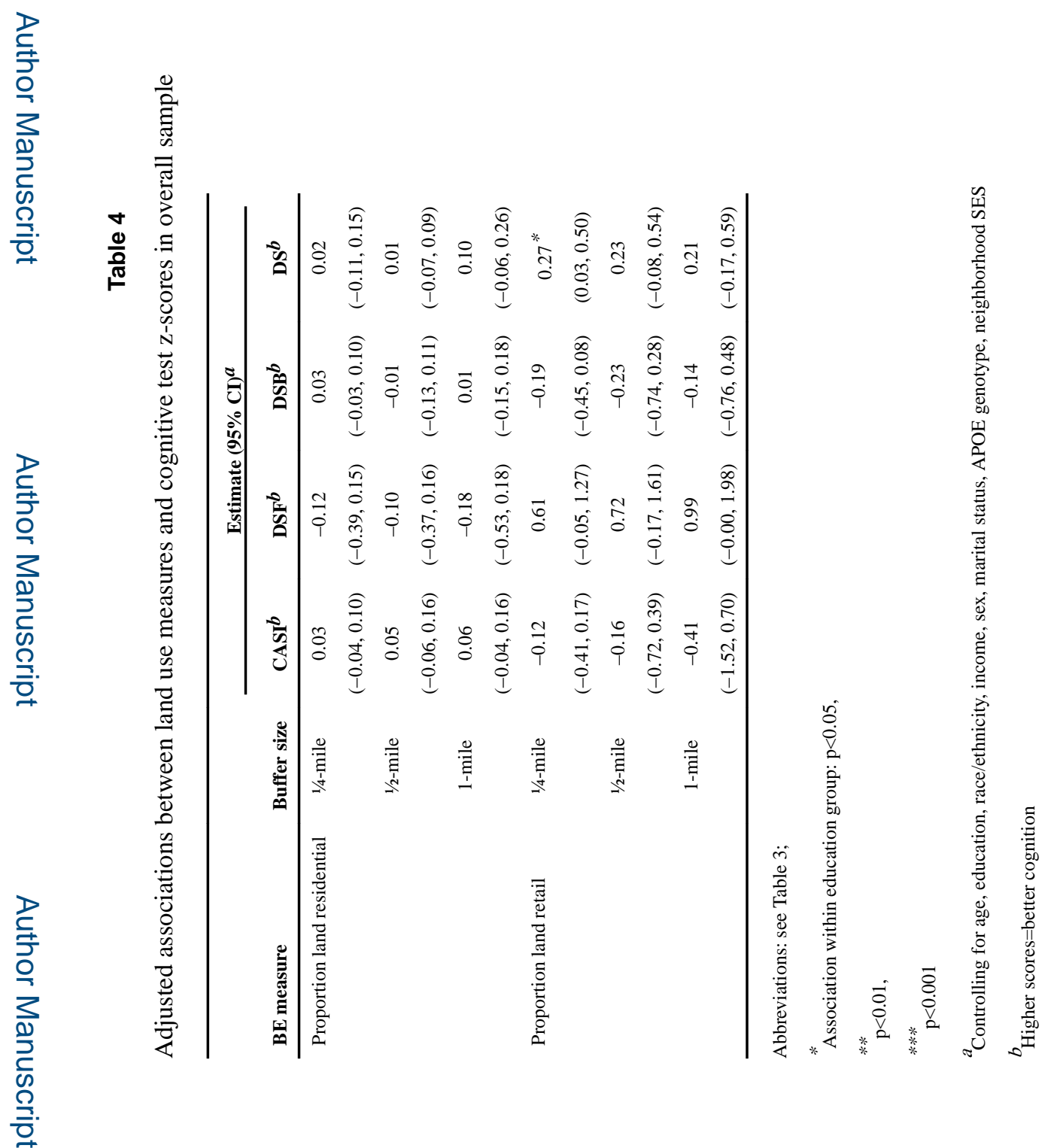

Soc Sci Med. Author manuscript; available in PMC 2019 March 01. 


\section{롤 \\ 골}

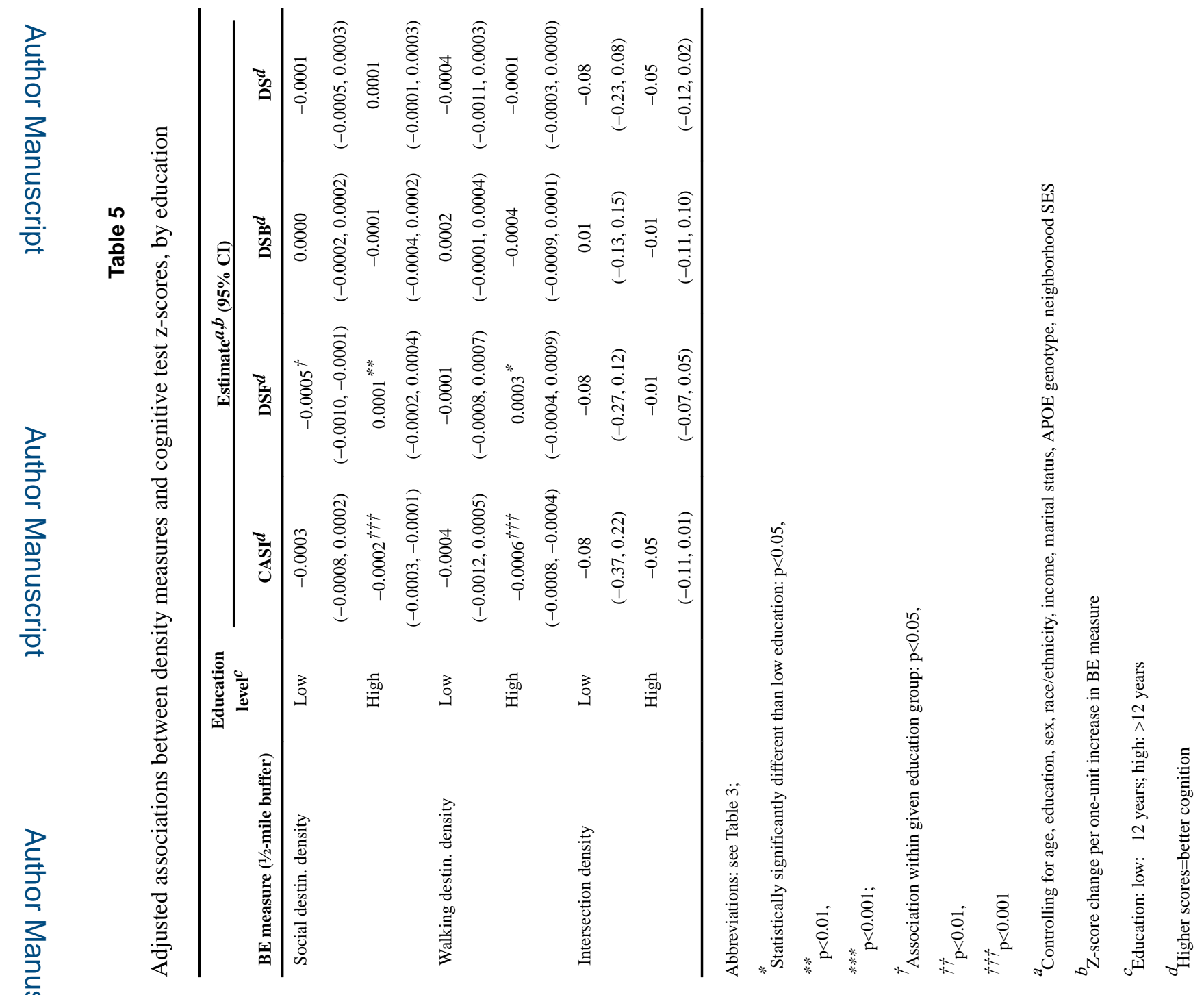




\section{롤 \\ 길}

로을



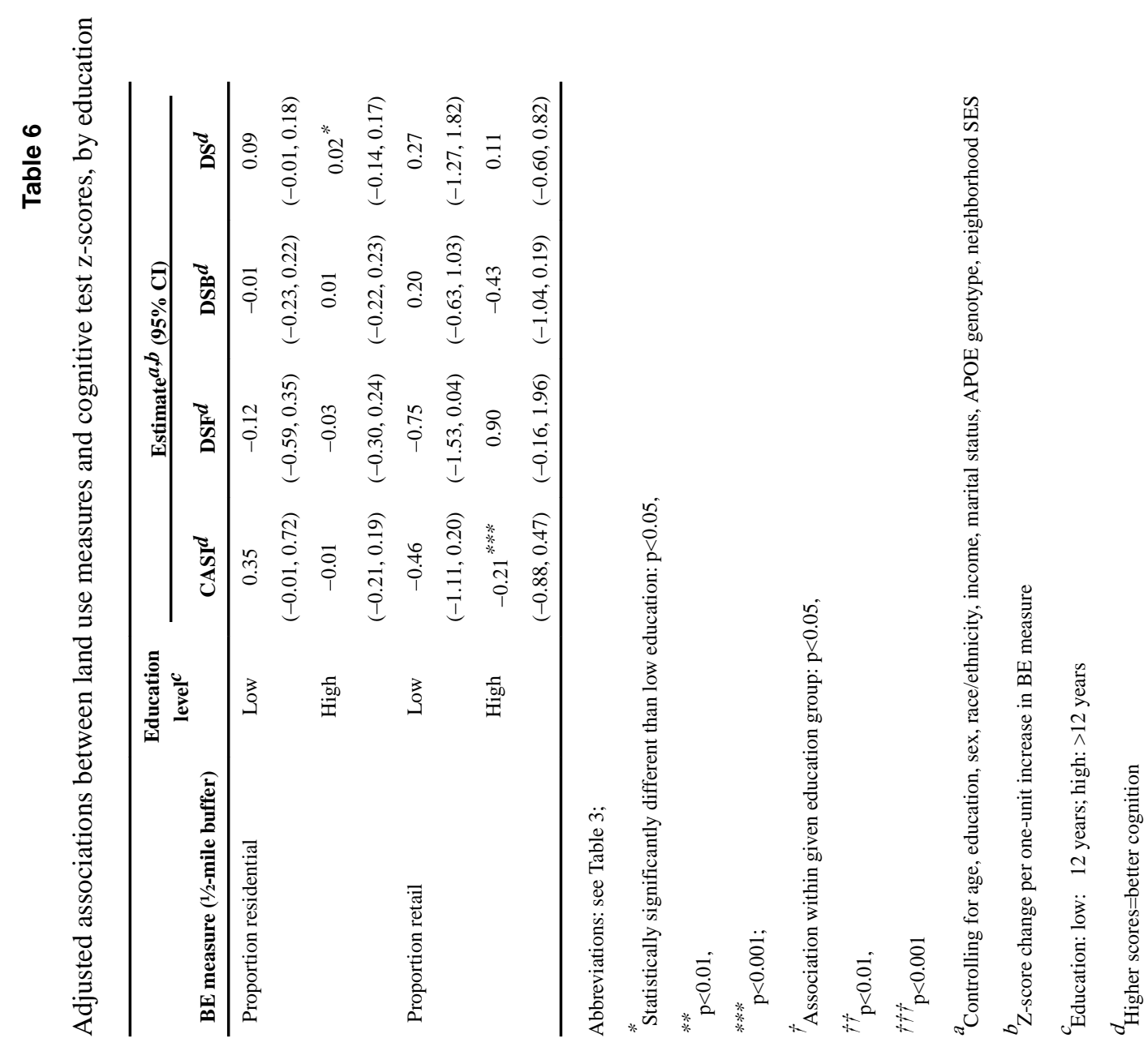

Soc Sci Med. Author manuscript; available in PMC 2019 March 01. 


\section{를 \\ ํㅗㄹ}

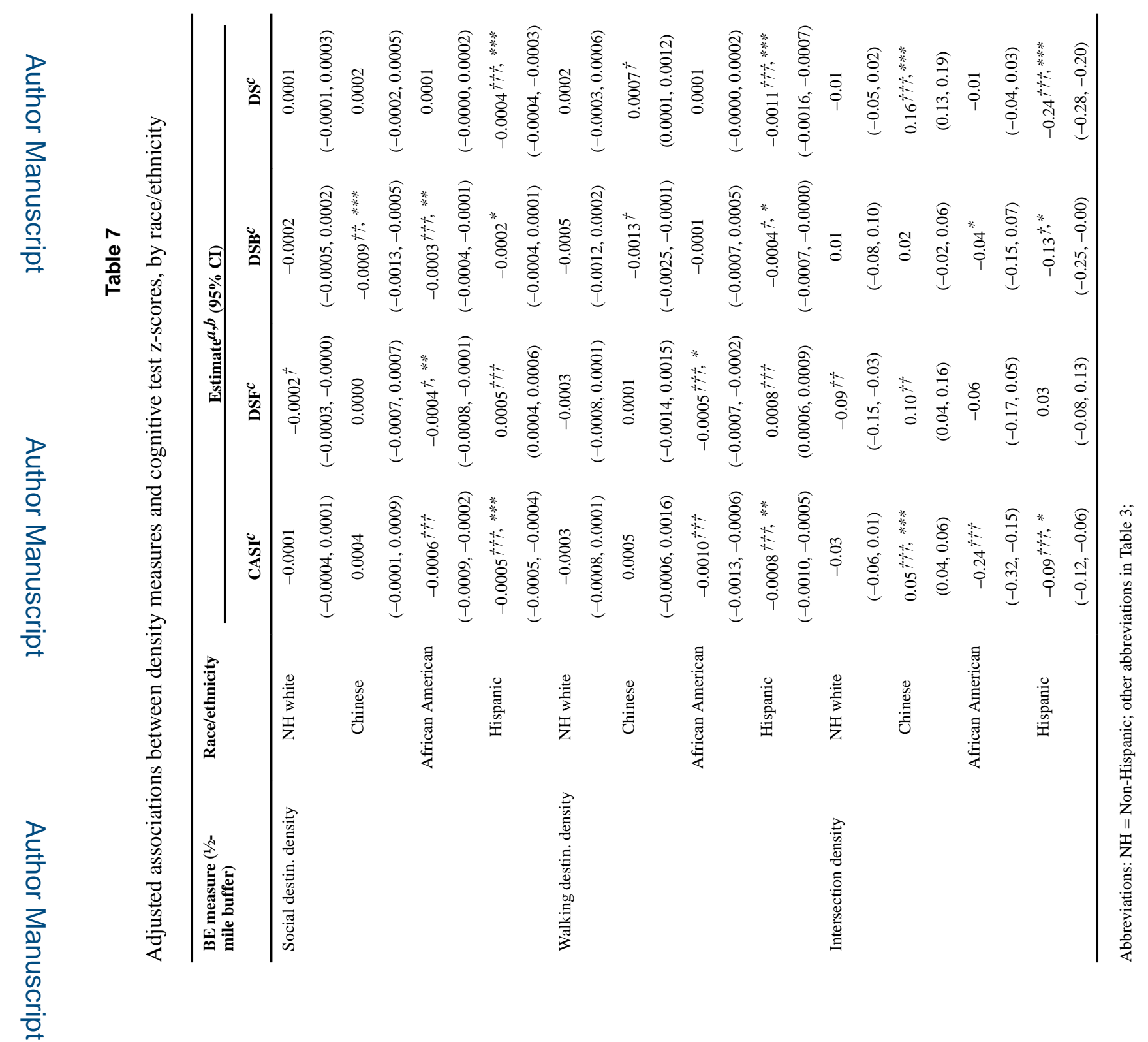

Soc Sci Med. Author manuscript; available in PMC 2019 March 01. 

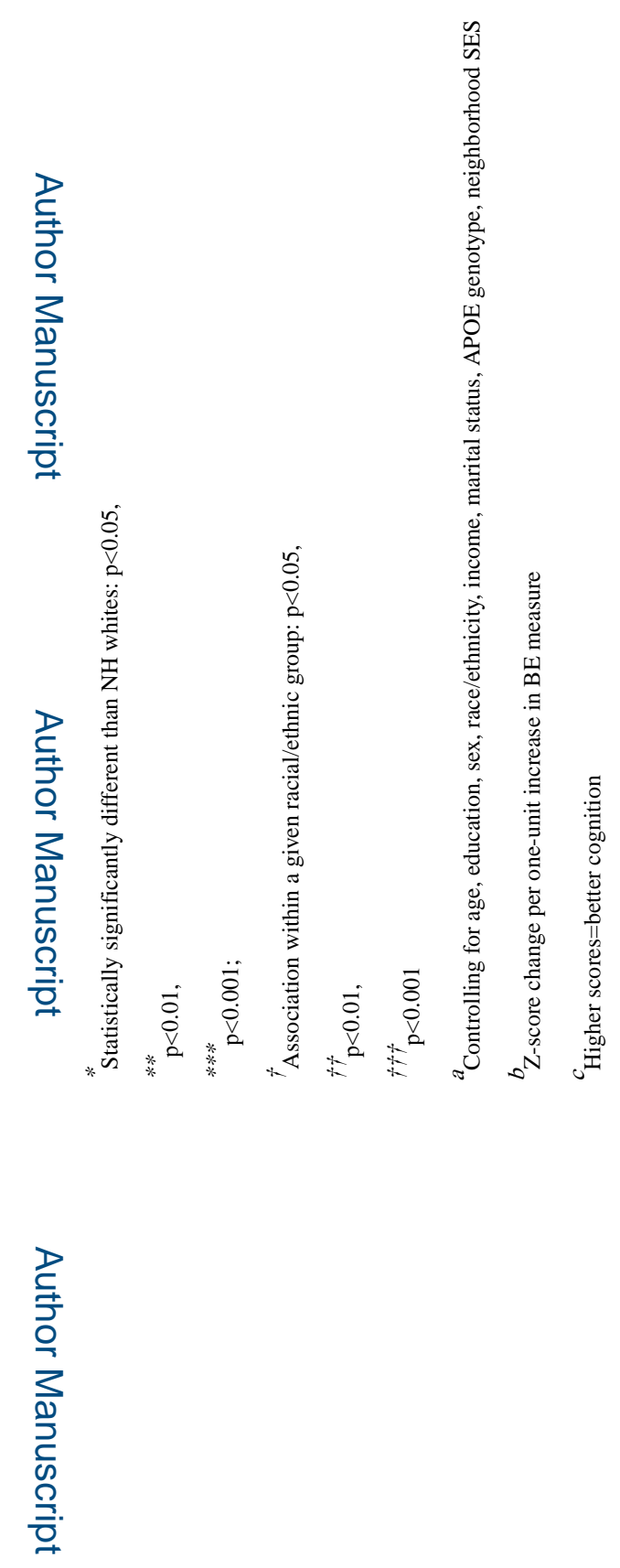

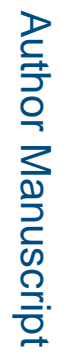

Soc Sci Med. Author manuscript; available in PMC 2019 March 01. 


\section{롤 \\ 골}

을

ב

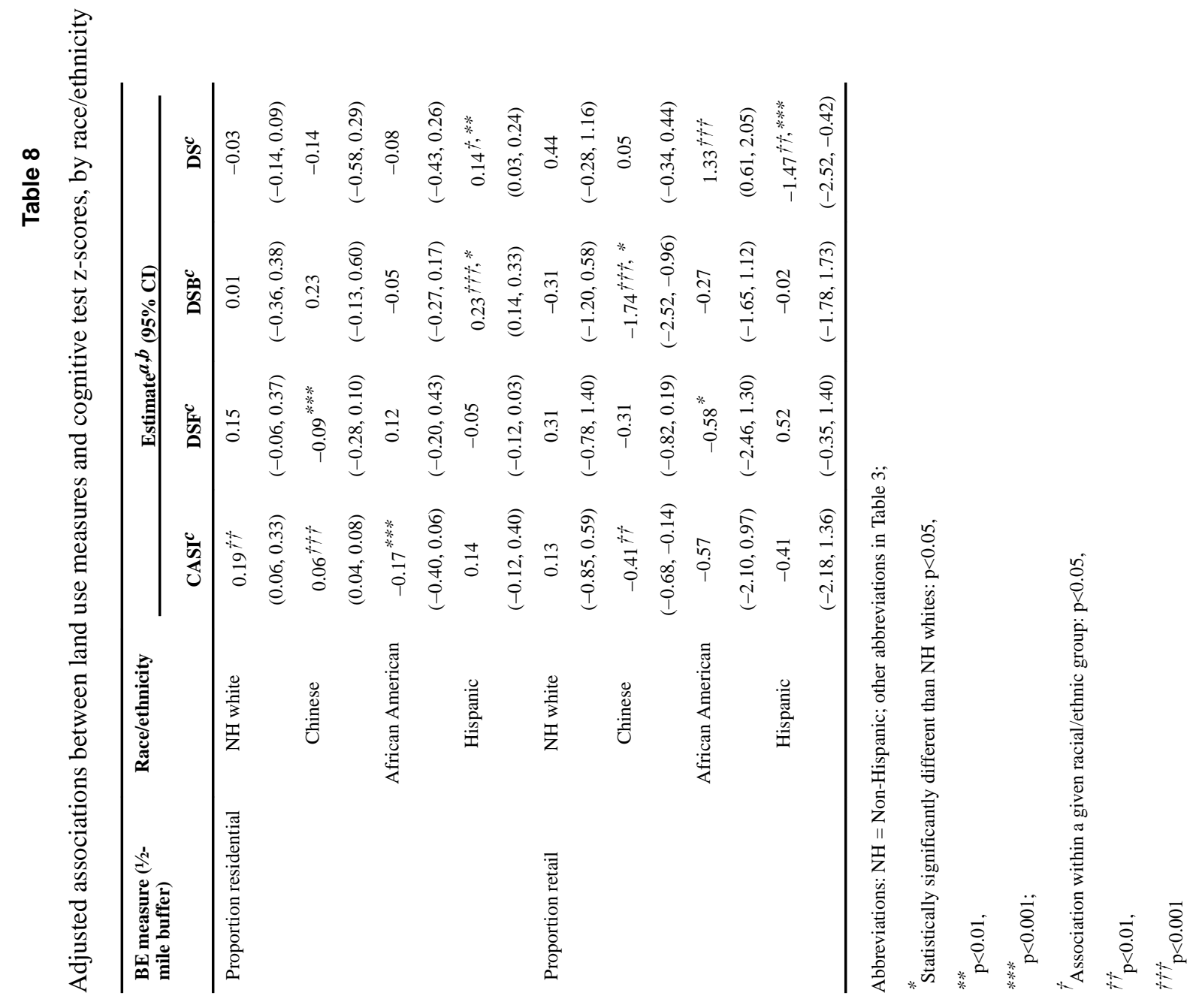



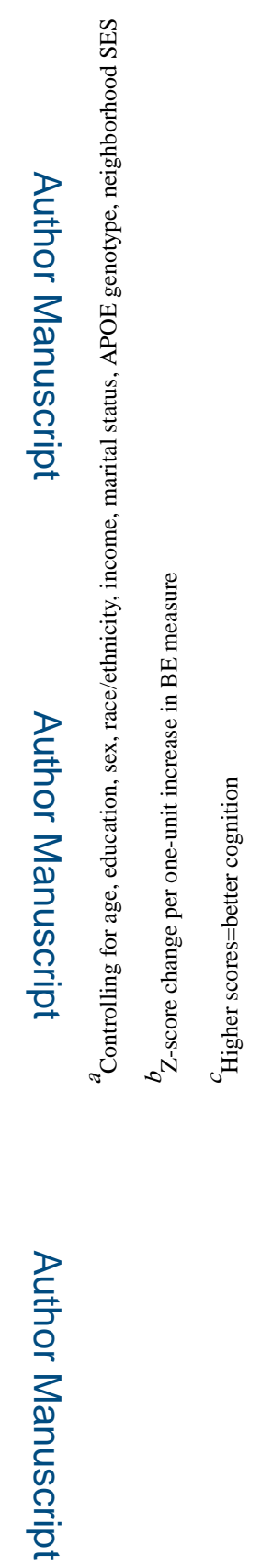

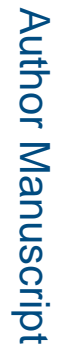

Soc Sci Med. Author manuscript; available in PMC 2019 March 01. 